\title{
Complejización de un modelo para el estudio de la resiliencia ambiental
}

\author{
Cruz García Lirios- Francisco Rubén Sandoval Vázquez- Bertha Leticia Rivera Varela- \\ Gerardo Arturo Limón Domínguez \\ Universidad Nacional Autónoma de México
}

\section{Resumen}

La resiliencia ambiental, vista como una respuesta sistemática de fuerzas que pretenden establecer el equilibrio en un campo o escenario deteriorado por el cambio climático, es el propósito del estudio. La discusión de sus modelos y sobre todo de relaciones entre sus elementos es el objetivo central de un trabajo documental que seleccionó información a partir de criterios de búsqueda de información en repositorios como Dialnet, Latindex y Redalyc. Se advierten matrices teóricas, conceptuales y empíricas desde las que es posible complejizar escenarios de intervención para el Trabajo Social.

\section{Palabras clave}

Cambio climático, salud pública, resiliencia ambiental, trabajo social, intervención 


\title{
Specification of a model for the study of environmental resilience
}

\begin{abstract}
Environmental resilience, often seen as a systematic response of forces seeking to establish equilibrium in a field or scenario deteriorated by climate change, is the purpose of the study. The discussion of its models and especially of relations between its elements is the central objective of a documentary work that selected information from criteria of search of information in repositories like Dialnet, Latindex and Redalyc. There are theoretical, conceptual and empirical matrices from which it is possible to anticipate intervention scenarios for Social Work.
\end{abstract}

\section{Keywords}

Climate change, public health, environmental resilience, social work, intervention

\section{Introducción}

La resiliencia entendida como un estilo de vida de dedicación, funcional, estable y vigoroso (Fernández y Crespo, 2011: p. 23) ha sido ampliamente utilizada por la psicología en la explicación de los efectos del cambio climático sobre la seguridad y la salud pública ambiental. En este proceso, la resiliencia es resultado de la mediación de factores organizacionales y psicológicos en el filtrado de las demandas sobre los recursos. De este modo, la resiliencia ambiental refiere a la capacidad de un sistema de absorber perturbaciones (Urquiza, 2015: p. 24). En ese sentido, el objetivo del presente trabajo es mapear, problematizar y complejizar un modelo para el estudio de éste fenómeno, considerando una selección documental de fuentes indexadas a Dialnet, Latindex y Redalyc, correspondientes al periodo que va de 2010 a 2017, así como la relación entre cambio climático, salud pública y resiliencia ambiental.

El proyecto, se inscribe en la división de ciencias sociales, disciplina de Trabajo Social, área de estudios documentales, pero incluye elementos de otras disciplinas de la misma división como la psicología, la economía, la antropología, la pedagogía y la administración. El proyecto fue financiado por la Universidad Nacional Autónoma de México, Dirección 
General de Asuntos del Personal Académico, Programa de Apoyo a Proyectos de Investigación e Innovación Tecnológica, número de registro IN305516.

\section{Teoría de la Resiliencia Ambiental}

En el marco de las Cumbres de la Tierra, las políticas de salud pública y las estrategias de resiliencia ambiental, la teoría cobra una especial relevancia ya que, a medida que los desastres naturales, catástrofes atmosféricas y crisis ecológicas se intensifican, la resiliencia ambiental se exacerba, principalmente en comunidades, barrios y localidades periféricas al desarrollo económico (Carreón, 2016).

En tal sentido, el estudio de la resiliencia ambiental se origina a partir de la incertidumbre y el riesgo que supone la inconmensurabilidad e impredecibilidad de los eventos que atañen a la salud comunitaria. El auge que ha cobrado el cambio climático en las Cumbres de la Tierra no sólo ha incidido sobre la agenda de los Estados y sus políticas públicas, sino además en sus estrategias de protección civil (Carreón, 2013).

La teoría de la resiliencia ambiental, en sus orígenes, estuvo encorsetada a la medición del riesgo en sus dimensiones objetivas y subjetivas, pero fue hasta que ésta matriz teórica alcanzó su plenitud cuando fusionó ambas dimensiones para dar cuenta de los efectos del cambio climático sobre el comportamiento humano, principalmente el cooperativismo y la solidaridad en las comunidades y barrios periféricos (García et al., 2016).

En un sentido objetivo, la teoría de la resiliencia ambiental apostó por el cálculo y predicción de la toma de decisiones ante los eventos contingentes. Se trató de una postura en la que se desarrolló un tecnicismo que determinó las políticas públicas de prevención y atención a población afectada (Carreón et al., 2015).

Las premisas centrales de la naciente teoría de la resiliencia ambiental se forjaron en los planteamientos epidemiológicos de salud pública en los que prevalecen los modelos de propagación de enfermedades y predador presa. El primero advertía sobre la interdependencia y vulnerabilidad de los sistemas de protección civil ante un evento inusitado, pero el modelo limitaba el análisis de factores externos al sistema entre los que se 
encontraba el cambio climático. Es así como bajo una lógica de exponentes y logaritmos, el modelo de propagación de enfermedades cedió su lugar al modelo del predador y la presa (García et al., 2012).

Si la propagación de enfermedades supone un estado de desesperanza de los individuos, esperando que las enfermedades aniquilen a sus futuras generaciones, el modelo de la presa y el predador pone de manifiesto que el entorno no es el único factor de destrucción de una generación o civilización, sino es la misma competencia por la escasez de los recursos la que obliga a los humanos a eliminarse unos con otros (García et al., 2017).

A partir de ambas propuestas, el modelo de dilema de los comunes llamo la atención por su anticipación catastrofista de considerar a los recursos ya no como públicos o privados, sino como comunes. El dilema de los comunes exageró sus pronósticos al deducir que la competencia genera la eliminación de los adversarios por la obtención y control del bien común (Carreón et al., 2016).

El modelo de dilema de los comunes no alcanzó a observar la cooperación solidaria entre comunidades que comparten recursos y que más allá de encasillarse en la competencia, las especies desarrollan sistemas solidarios (García et al., 2014).

La teoría de la resiliencia ambiental fue influida por la teoría de los bienes comunes al asumir que la cooperación y la solidaridad prevalecen sobre cualquier otra manifestación individual y social ante la escasez de los recursos. De este modo, el desarrollo de modelos de control externo e interno contribuyeron a mostrar que la organización social se gesta cuando existe una percepción de control sobre los recursos, pero cuando se genera la desconfianza, entonces lejos de observarse formas cooperativas o de competencia se miran más bien formas de indiferencia o desesperanza que serían opuestas a la solidaridad (García et al., 2016).

Es así como la teoría de la resiliencia ambiental centró su interés en el emprendimiento y la innovación, pero ya no como resultado de la opresión política o social, sino como efecto del cambio climático, la escasez de los recursos y el deterioro de la salud pública. De este modo, la teoría explica el proceso arduo de una comunidad o localidad ante los desastres naturales, la competencia por los recursos, la cooperación, el emprendimiento y la innovación como 
manifestaciones particulares y globales de individuos y grupos que buscan emanciparse o reivindicarse ante los límites del crecimiento que impone la naturaleza. (Carreón, 2016).

\section{Estudios de la Resiliencia Ambiental}

Los estudios psicológicos de la resiliencia ambiental han especificado modelos sin incluir variables sociopolíticas como la intención de voto a favor de propuestas verdes, el emprendimiento social o el posmaterialismo social (Carreón, 2013).

No obstante que los modelos especificados centran su interés en las comunidades, su relación con el Estado permitiría observar los niveles de desarrollo local sustentable a partir de las políticas de fomento empresarial y emprendimiento social, aunque son los medios de comunicación los que al establecer temas en la agenda pública determinan las representaciones sociales de la ciudadanía con respecto a sus autoridades y en función de éstos símbolos se generan las políticas públicas de asistencia social (García et al., 2012).

Los modelos especificados tampoco incluyen las relaciones de dependencia entre variables cognitivas tales como creencias, actitudes e intenciones determinantes del comportamiento pro ambiental aún y cuando estas trayectorias explican las políticas de tarifas de los servicios municipales, los modelos especificados sólo anticipan comportamientos más que escenarios de acción colectiva y movilización social como reflejo de los conflictos entre autoridades y usuarios (Carreón et al., 2015).

La inclusión de las variables sociopolíticas, cognitivas y comunitarias explicaría el isomorfismo relativo a la gobernanza como objeto de estudio de la complejidad ambiental. A diferencia del estrés o la resiliencia que suponen reacciones de las comunidades ante los eventos de riesgo ambiental, la gobernanza es indicativa de un proceso en el que los recursos

y discursos de orden neguentrópico contra-restan los efectos de la identidad entrópica. Esto es así porque el capital humano parece estar vinculado a la producción de innovaciones que reduzcan la hipermetropía y enaltezcan la cooperación (Carreón, 2013).

Ante los desastres naturales, la comunicación de riesgos sería otra variable a incluir en el modelo para el estudio del estrés y la resiliencia, ya que, un aumento de los eventos de riesgo 
supone un mayor estrés. Por consiguiente, la formación del capital humano estaría en función de la anticipación de los riesgos y no posterior a los desastres y catástrofes naturales (García et al., 2015).

Incluso, en lugar de la anticipación de los riesgos, se desarrolla una indiferencia conocida como hipermetropía que minimiza el alcance de los desastres naturales en las localidades y cuando son percibidas las catástrofes como cercanas, entonces se maximizan sus efectos responsabilizando al Estado de la seguridad ambiental (García et al., 2012).

Por lo tanto, un modelo explicativo y anticipativo de la gobernanza no sólo incluiría las variables sociopolíticas y psicológicas, sino además anticiparía el surgimiento de una agenda que legitime la rectoría del Estado en materia de seguridad ambiental y reduzca a su mínima expresión a la participación ciudadana en cuanto a su identidad neguentrópica (Carreón, 2013).

En la medida en que los riesgos, desastres y estrés ambientales se intensifican propician la emergencia de identidades entrópicas y neguentrópicas que determinarán la resiliencia de las comunidades ante el desabastecimiento de recursos naturales y servicios municipales (García et al., 2014).

La psicología de la resiliencia ambiental ha establecido cuatro factores -autorregulación, disipación, adaptabilidad y dinamismo- que corresponden a una estructura de relaciones posibles en explicación de la complejidad organizacional (Carreón et al., 2015).

Sin embargo, los estudios de la complejidad organizacional y de las redes neuronales advierten que los procesos de aprendizaje suponen una instancia latente que correspondería con una instancia manifiesta. En este sentido, la complejidad oculta referiría a los procesos de aprendizaje y conocimiento implícito que sólo la sistematización laboral concede, pero sería una complejidad manifiesta si tal procesamiento de información, elaboración e implementación de estrategias se establecen en las tareas (García et al., 2016).

De este modo, los objetivos, metas y logros son parte de un proceso informacional y comunicativo desde el que la complejidad es aprendida. Tal proceso es observable en la 
autoregulación, la disipación, la adaptación y el dinamismo en torno a las oportunidades y capacidades (García et al., 2017).

La psicología de la resiliencia ambiental ha establecido escenarios de desarrollo comunitario con respecto al emprendimiento social ya no sólo como un efecto de las políticas de microfinanciamiento, sino como una iniciativa comunitaria asociada a las expectativas de caficultores (Carreón, 2016).

En este sentido, los escenarios posibles son:

Alianzas, micro-financiamiento y desarrollo endógeno. Radica en las fusiones entre empresas locales y trasnacionales con la finalidad de aprovechar el micro-financiamiento del Estado y activar la economía local mediante la comercialización en empresas vinculadas al desarrollo regional. En contraste con el modelo neoliberal en el que las trasnacionales determinan la comercialización de productos, en este escenario son las mipymes las que definen las estrategias de producción-venta al menudeo y la eventual exportación del producto (García et al., 2016).

Apoyos, innovación y desarrollo de mipymes. Estriba en becas y apoyos a grupos vulnerables que ahorran y utilizan el dinero con base en la propuesta de nuevos servicios y productos. En relación con el modelo de micro-financiamiento en el cual los beneficiarios se organizan en cooperativas para abonar los adeudos y continuar con los apoyos a otros sectores, en este escenario la innovación permite la subsistencia de quienes se dedican al comercio informal (García et al., 2015).

Identidad, micro-financiamiento y desarrollo comunitario. Consistiría en el establecimiento de alianzas, motivadas por el sentido de pertenencia al lugar de origen, entre exmigrantes y oriundos, pero a diferencia del modelo de bienes comunes en los que los actores, económicos, políticos y sociales deciden el modo de producción, es la comunidad que, con base en diálogo de saberes, establece los acuerdos y las corresponsabilidades (García et al., 2014).

Tales escenarios y factores podrían adquirir mayor relevancia si se considera que la comercialización de productos y servicios es una actividad económica impulsada por 
políticas de fomento empresarial y micro financiamiento, así como parte de los usos y costumbres sociales y comunitarios de la localidad de estudio (García et al., 2012).

Además, las condiciones ambientales también juegan un papel decisivo en las percepciones de emprendimiento en general de productos y servicios agrícolas y las percepciones de oportunidad, así como las capacidades impulsadas por las políticas de fomento empresarial y micro-financiamiento al emprendimiento social (Carreón et al., 2015).

Por consiguiente, la especificación de un modelo explique las particularidades de las localidades emprendedoras es menester, ya que, si bien el emprendimiento supone una estructura de percepciones, decisiones y estrategias generales, el éxito de los proyectos depende de la especificidad local (Carreón et al., 2014).

Las características de cada sector obligan a un análisis minucioso acerca de los usos y costumbres que hacen posible la subsistencia mediante el emprendimiento social luego ser incentivado por el micro-financiamiento, la formación y capacitación son dos factores a considerar en el desarrollo endógeno. La exploración de valores, percepciones y creencias permitirá explicar y anticipar escenarios de crisis ambiental, económica, política y social, pero también de resiliencia y emprendimiento social (García et al., 2017).

El diagnóstico de los factores e indicadores permitiría una intervención más actualizada y específica del Trabajo Social ya que, en la historia de la disciplina, el Estado ha determinado sus modelos de intervención, pero en una localidad pujante ha permitido la emergencia de sectores emprendedores de la sociedad civil, la intervención cobra un sentido diferente (García et al., 2016).

Por consiguiente, en cada uno de los tres escenarios, la intervención adquiere; a) un sentido mediador donde se asume que el Trabajo social es informante de las necesidades y expectativas de grupos emprendedores, excluidos o migrantes con respecto a la comercialización del café y b) un sentido moderador en el que los profesionistas del Trabajo Social se involucran en el emprendimiento social y generar iniciativas para incidir en las políticas de micro-financiamiento (García et al., 2015). 
Se trata de tres fundamentos, a saber:

Escenario de mayor competencia \Alianzas, micro-financiamiento y desarrollo endógeno predomina el emprendimiento de mipymes establecidas y reguladas por las leyes del mercado y los ordenamientos del Estado, confinando a los profesionistas del Trabajo Social a transferir los valores y normas de una cultura de emprendimiento tradicional donde las oportunidades y las capacidades son atribuciones exclusivas de talentos y líderes que compiten por la asignación de los recursos (Carreón et al., 2014).

Escenario de asignación discrecional \Apoyos, innovación y desarrollo de mipymes」 consiste en la determinación de los presupuestos y montos por parte de los actores gubernamentales con respecto a los actores económicos y sociales. La función del profesionista del Trabajo Social permanece confinada a brindar información del contexto local para justificar la designación de micro-financiamientos (Carreón, 2013).

Escenario de emprendimiento social \Identidad, micro-financiamiento y desarrollo comunitario J se trata de sentidos de apego al lugar de origen que determinan el retorno de exmigrantes y las alianzas con emprendedores oriundos. En este proceso, la contribución del profesionista del Trabajo Social es fundamental ya que, en la medida en que un diagnóstico se aproxime a la cooperación y la solidaridad, genera iniciativas de gestión y administración de recursos que determinarán el desarrollo local sin importar el micro-financiamiento del Estado (Carreón, 2016).

\section{Complejización de un modelo para el estudio de la resiliencia ambiental}

Un modelo necesariamente supone una especificación de las trayectorias de relaciones de dependencia entre variables que explican consistentemente un fenómeno ampliamente observado en el estado del conocimiento y la revisión de la literatura. Pues bien, complejizar un modelo consiste en algo opuesto a la especificación. Se trata de discutir los límites de ésta con la finalidad de observar relaciones complejas como la auto-organización, la recursividad, la emergencia o la fractalidad entre otros procesos complejos (García et al., 2017). 
De este modo, la complejización del modelo se reduce a un solo eje hipotético, pero no en el sentido de simplificarlo sino más bien se busca observar su impredecibilidad si se asociación algunos elementos, bajo el supuesto de una separación con los demás factores (véase Figura $1)$.

Figura 1. Complejización de un modelo para el estudio de la resiliencia ambiental

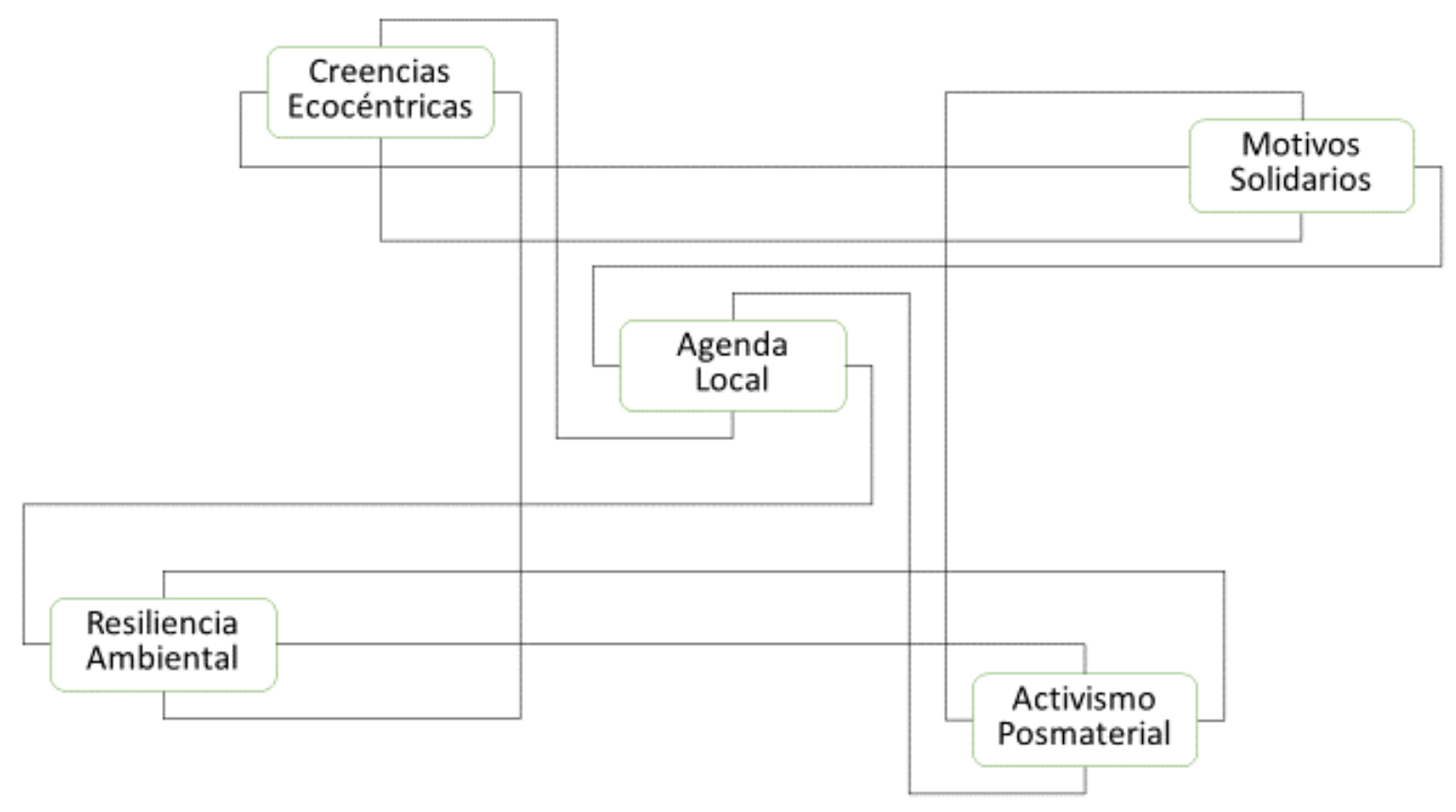

Fuente: Elaboración propia

Es así como, el proceso que va del cambio climático, cumbres de la tierra, programas de protección civil, prevención o reducción de la huella ambiental, la atención a la salud pública y la resiliencia ambiental como indicador de una acción colectiva y movilización social cultivada mediante el activismo ecológico para ahorrar los recursos, es observable en unos cuantos elementos que se reproducen en cada una de las relaciones esgrimidas.

Es el caso del establecimiento de una agenda local que consiste en la repetición sistemática de temas y ejes de discusión con la finalidad de persuadir a audiencias o disuadirlas de sus creencias ecocéntricas, motivos solidarios, resiliencia ambiental y activismo posmaterial. Es decir, la observación de estos cinco elementos citados devela una estructura emergente en cada una de las relaciones del proceso que va del cambio climático al ahorro de recursos. 


\section{Consideraciones finales}

El aporte del presente trabajo al estado del conocimiento estriba en la complejización de un modelo para el estudio de la resiliencia ambiental como factor emergente, recursivo, fractal y borroso, pero limitado a la literatura consultada y los criterios de búsqueda y selección informativa.

Se recomienda: 1) llevar a cabo una revisión de la literatura incluyendo los conceptos de "complejidad", “emergencia", “disipación”, fractalidad", "recursividad", “autoorganización" y "caos" para poder establecer una mayor complejización y desarrollar métodos, técnicas y parámetros que permitan el estudio de la resiliencia ambiental como efecto y causa de un escenario de abundancia y escasez de recursos, así como de competencia y solidaridad entre las especies; 2) modelar sus relaciones enfatizando sus posibilidades de vinculación entre las variables a fin de poder desarrollar los supuestos teóricos que darán origen a nuevos conceptos desde los que se explicará el fenómeno; 3) anticipar y proponer estrategias de intervención asumiendo los imponderables de las relaciones posibles, aunque esperando la exacerbación de estilos de vida desfavorables y favorables a los cambios ambientales y de salud pública.

\section{Referencias}

Carreón J. (2016). Desarrollo humano: Gobernanza y emprendimiento social. México: UNAM-ENTS

Carreón, J. (2013). Discursos en torno a la migración laboral, el retorno y la reincersión social a partir de la identidad grupal en Xilitla, micro-región de la huasteca potosina (Mëxico). En L. Cano (coord.). Pobreza y desigualdad social. Retos para la reconfiguración de la política social. (pp. 153-174). México: UNAM-ENTS

Carreón, J., De la Cruz, P. I. y De los Santos, M. (2015). La administración de temores sociales. Contrastes de un modelo de percepción de la inseguridad en México. Ehquidad, 4, 31-60 
Carreón, J., Hernández, J., Quintero, M. L., García, C. y Mejía, S. (2016). Redes de conocimiento en torno a la complejidad organizacional: aprendizaje de la autoregulación, disipación, adaptabilidad y dinamismo ante los cambios. Prospectiva, 2 (2), 57-70

Fernández, V. y Crespo, M. (2011). Resiliencia, personalidad resistente y crecimiento en cuidadores de personas con demencia en el entorno familiar. Ciencia y Salud, 22 (1), $21-40$

García, C., Carreón, J. y Hernández, J. (2017). Límites de los modelos de salud ocupacional. Estudio de la adhesión al tratamiento del asma en trabajadores migrantes adultos mayores del Estado de México. Visión Gerencial, 16, 103-118

García, C., Carreón, J., Hernández, J. y Salinas. (2016). Gobernanza de los actores y redes de innovación tecnológica. En M. L. Quintero, Sales, J. y Velázquez, E. B: (coord.). Innovación y tecnología. Retos para su aplicación práctica en las empresas. (pp. 7994). México: Porrúa-UAEMEX UAP Nezahualcóyotl

García, C., Carreón, J., Hernández, J., Aguilar, J. A., Rosas, F. J. y Bustos, J. M. (2015). Diferencias de fiabilidad ante riesgo, incertidumbre y conflicto entre caficultores en Xilitla, México. Eureka, 12 (1), 73-93

García, C., Carreón, J., Hernández, J., Carbajal, C., Quintero, M. L., Sandoval, F. R. y Valdés, O. (2016). Incidencia de las políticas de micro-financiamiento sobre la percepción de emprendimiento caficultor e implicaciones para el Trabajo Social. Equidad, 6, 11-36

García, C., Carreón, J., Hernández, J., Montero, M. y Bustos, J. M: (2012). Sistemas de complejidad política. Trabajo Social Hoy, 65, 39-48

García, C., Carreón, J., Mecalco, J., Hernández, J., Bautista, M. y Méndez, A. (2014). Sistemas políticos complejos: Implicaciones para la seguridad pública sustentable. Acciones e Investigaciones Sociales, 34,186-216 
García, C., Carreón, J., Sandoval, F. R., Bustos, J. M. y Aguilar, J. A. (2016). Estructura de la cultura laboral en una institución de promoción de salud pública. Arequipa, 6 (1), 291-304

Urquiza, A. y Cadenas, H. (2015). Sistemas socio-ecológicos: Elementos teóricos y conceptuales para la discusión en torno a la vulnerabilidad hídrica. Orda, 208, 1-33 